\title{
Predictive ability of pharyngeal inlet angle for the occurrence of postoperative dysphagia after occipitocervical fusion
}

Lin-nan Wang, Bo-wen Hu, Yue-ming Song, Li-min Liu, Chun-guang Zhou, Lei Wang and Xi Yang*

\begin{abstract}
Background: PIA has been proven to be a predictor for postoperative dysphagia in patients who undergo occipitospinal fusion. However, its predictive effect for postoperative dysphagia in patients who undergo OCF is unknown. The aim of this study was to evaluate the predictive ability of the pharyngeal inlet angle (PIA) for the occurrence of postoperative dysphagia in patients who undergo occipitocervical fusion (OCF).

Methods: Between 2010 and 2018, 98 patients who had undergone OCF were enrolled and reviewed. Patients were divided into two groups according to the presence of postoperative dysphagia. Radiographic parameters, including the atlas-dens interval (ADI), O-C2 angle (O-C2a), occipital and external acoustic meatus to axis angle (OEAa), C2 tilting angle (C2Ta), C2-7 angle (C2-7a), PIA and narrowest oropharyngeal airway space (nPAS), were measured and compared. Simple linear regression and multiple regression analysis were used to evaluate the radiographic predictors for dysphagia. In addition, we used PIA $=90^{\circ}$ as a threshold to analyze its effect on predicting dysphagia.
\end{abstract}

Results: Of the 98 patients, 26 exhibited postoperative dysphagia. Preoperatively, PIA in the dysphagia group was significantly higher than that in the nondysphagia group. We detected that O-C2a, O-EAa, PIA and nPAS all decreased sharply in the dysphagia group but increased slightly in the nondysphagia group. The changes were all significant. Through regression analyses, we found that PIA had a similar predictive effect as O-EAa for postoperative dysphagia and changes in nPAS. Additionally, patients with an increasing PIA exhibited no dysphagia, and the sensitivity of PIA $<90^{\circ}$ in predicting dysphagia reached $88.5 \%$.

Conclusions: PIA could be used as a predictor for postoperative dysphagia in patients undergoing OCF. Adjusting a PIA level higher than the preoperative PIA level could avoid dysphagia. For those who inevitably had decreasing PIA, preserving intraoperative PIA over $90^{\circ}$ would help avert postoperative dysphagia.

Trial registration: This trial has been registered in the Medical Ethics Committee of West China Hospital, Sichuan University. The registration number is 762 and the date of registration is Sep. 9 th, 2019.

Keywords: Occipitocervical fusion, Postoperative dysphagia, O-EAa, PIA, Prediction

\footnotetext{
*Correspondence: formosa88@163.com

Department of Orthopedics Surgery and Orthopaedics Research Institute, West China Hospital, Sichuan University, No. 37 GuoXue Road, 610041

Chengdu, Sichuan, China
}

(c) The Author(s). 2021 Open Access This article is licensed under a Creative Commons Attribution 4.0 International License, which permits use, sharing, adaptation, distribution and reproduction in any medium or format, as long as you give appropriate credit to the original author(s) and the source, provide a link to the Creative Commons licence, and indicate if changes were made. The images or other third party material in this article are included in the article's Creative Commons licence, unless indicated otherwise in a credit line to the material. If material is not included in the article's Creative Commons licence and your intended use is not permitted by statutory regulation or exceeds the permitted use, you will need to obtain permission directly from the copyright holder. To view a copy of this licence, visit http://creativecommons.org/licenses/by/4.0/ The Creative Commons Public Domain Dedication waiver (http://creativecommons.org/publicdomain/zero/1.0/) applies to the data made available in this article, unless otherwise stated in a credit line to the data. 


\section{Background}

Dysphagia is one of the most commonly seen complications after occipitocervical fusion (OCF), with an incidence ranging from 15.8-26.6\% [1-4]. Most of these patients have a protracted course, and dysphagia interferes with the quality of daily living [3-6]. For this reason, the relationship between postoperative dysphagia and changes in occipitocervical alignment has been studied by many researchers, and several predictors have been promoted $[1,3-5,7,8]$.

The O-C2 angle (O-C2a) was the most commonly used predictor in previous studies since it was first described in 2007 [9]. A significant reduction in O-C2a was correlated with a decrease in oropharyngeal space and postoperative dysphagia $[1,10,11]$. The newly promoted occipital and external acoustic meatus-to-axis angle (O-EAa) is the sum of the $\mathrm{O}-\mathrm{C} 2 \mathrm{a}$ and $\mathrm{C} 2$ tilting angles $(\mathrm{C} 2 \mathrm{Ta})$ and can reflect not only craniocervical junction alignment but also cranial transverse motion, thus compensating for the shortcomings of O-C2a [7]. In our previous study, we reviewed 109 OCF patients and found that O-EAa was superior to O$\mathrm{C} 2 \mathrm{a}$ in predicting decreases in oropharyngeal space and postoperative dysphagia [4]. However, during the measurement of O-EAa, we found it difficult to identify the external acoustic meatus in some patients.

The pharyngeal inlet angle (PIA) was promoted by Kaneyama in 2017 and was defined as the angle between McGregor's line and the line that links the center of the $\mathrm{C} 1$ anterior arch and the apex of cervical sagittal curvature [3]. These anatomical structures could be easily identified on intraoperative radiographic images. Previously, this angle has been proven to be a predictor for postoperative dysphagia in patients who undergo occipitospinal fusion [3]. Therefore, we conducted this study to evaluate the predictive effect of PIA for the development of postoperative dysphagia in patients who undergo OCF and to compare the predictive abilities of PIA and several other radiographic parameters.

\section{Methods}

In the present study, we reviewed the same batch of patients from April 2010 to June 2018 as in our previous study [4]. The inclusion criteria were as follows: (1) post-OCF patients; (2) a minimum follow-up of 12 months; and (3) complete preoperative and postoperative radiographic records. The exclusion criteria were as follows: (1) presence of preoperative dysphagia; (2) underwent transoral surgery or esophageal disease; (3) unable to evaluate postoperative swallowing conditions; and (4) unidentifiable anterior arch of $\mathrm{C} 1$ on lateral radiographic images.

\section{Radiographic measurements}

Preoperative and one month postoperative standing lateral cervical radiographic images in a neutral position with a horizontal gaze were used to measure the following radiographic parameters (Fig. 1):

Atlas-dens interval (ADI)- the distance between the back edge of the anterior arch of the atlas and the front edge of the odontoid process;

$\mathrm{O}-\mathrm{C} 2 \mathrm{a}$ - the angle between McGregor's line and the inferior end plate of $\mathrm{C} 2$;

O-EAa- the angle formed by McGregor's line and the line connecting the midpoint of the bilateral external acoustic meatus and the midpoint of the inferior end plate of C2 (EA line);

$\mathrm{C} 2 \mathrm{Ta}$ - the angle formed by the inferior endplate of $\mathrm{C} 2$ and the EA line;

C2-7 angle (C2-7a)- the angle between the inferior endplate of $\mathrm{C} 2$ and $\mathrm{C} 7$;

PIA- the angle between McGregor's line and the line that links the center of the $\mathrm{C} 1$ anterior arch and the apex of the cervical sagittal curvature;

Narrowest oropharyngeal airway space (nPAS)- the narrowest anterior-posterior diameter of the oropharynx between the tips of the uvula and epiglottis.

dADI, dO-C2a, dO-EAa, dC2Ta, dC2-7a, dPIA and dnPAS were calculated as the 1-month postoperative values minus the preoperative values. dnPAS\% was calculated as dnPAS/preoperative nPAS.

\section{Dysphagia assessment}

Inpatient medical records were reviewed to assess preoperative swallowing conditions. As in our previous study, the presence and duration of postoperative dysphagia were evaluated through inquiry by clinic or telephone interviews. The presence of postoperative dysphagia was defined as the occurrence of difficulty or taking extra effort in swallowing after surgery [12]. The grade of dysphagia was also evaluated for all patients during follow-up according to the dysphagia classification scale created by Bazaz et al. [13]. "Severe" dysphagia was defined as frequent difficulty swallowing with the majority of foods, "moderate" dysphagia was defined as occasional swallowing difficulty for specific foods, "mild" dysphagia was defined as rare episodes of dysphagia, and "none" was defined as no episodes of dysphagia. Patients with postoperative dysphagia less than one month duration was considered as side-effect caused by intubation and were considered as no occurrence of dysphagia.

\section{Statistical analysis}

Measurements were independently performed twice in a week by two authors. Reliability was evaluated by calculating the intraclass correlation coefficient. Data were expressed as the mean \pm standard deviation values. SPSS 23.0 (SPSS Inc, Chicago, Illinois) software was used to analyze the collected data. The paired Student's $t$ test and Mann-Whitney $U$ test were used to compare 


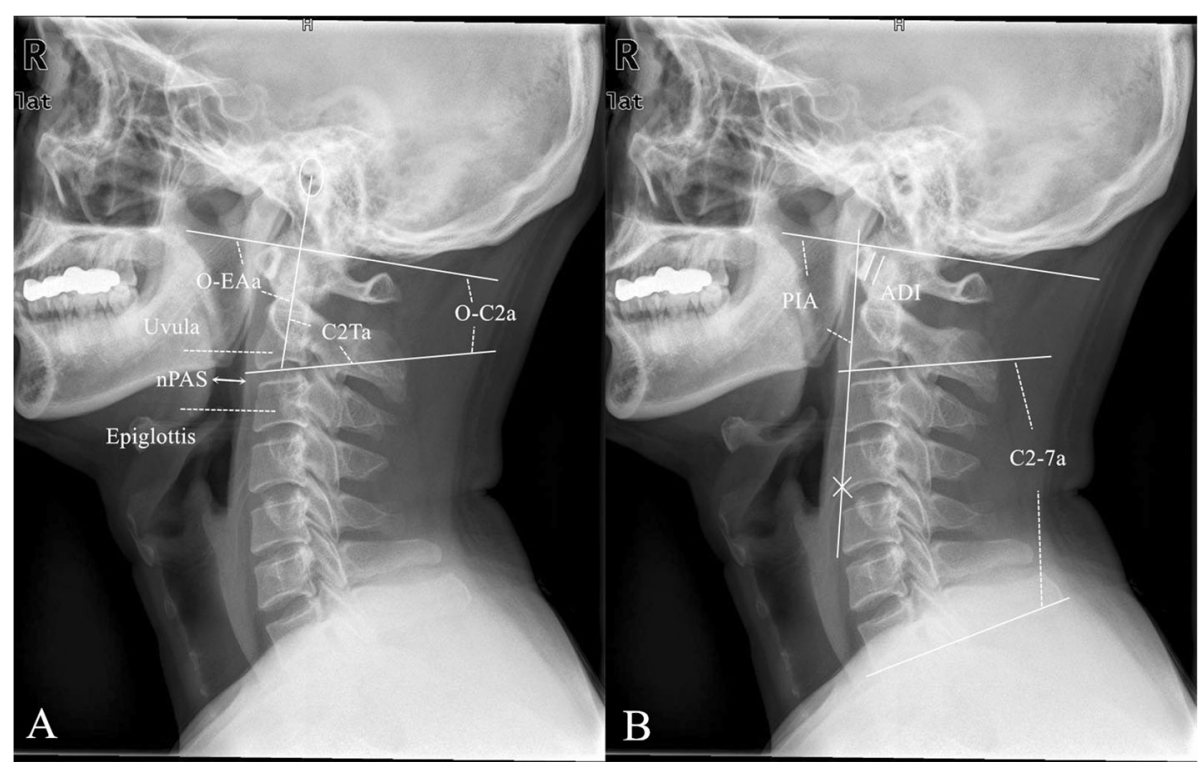

Fig. 1 Representation of radiographic measurements. a O-C2a is the angle between McGregor's line and the inferior endplate of the axis. O-EAa is the angle formed by McGregor's line and the line connecting the midpoint of the bilateral external acoustic meatus and the midpoint of the inferior end plate of C2 (EA line). C2Ta is the angle formed by the inferior endplate of the C2 and EA lines. nPAS is the narrowest anteriorposterior diameter of the oropharynx between the tips of the uvula and epiglottis. $\mathbf{b}$ ADI is the distance between the back edge of the anterior arch of the atlas and the front edge of the odontoid process. C2-7a is the angle between the inferior endplate of C2 and C7. PIA is the angle between McGregor's line and the line that links the center of the $\mathrm{C} 1$ anterior arch and the apex of cervical sagittal curvature

continuous variables. A simple linear regression was used to investigate the association between collected radiographic parameters and the occurrence of postoperative dysphagia. Previously, we proved a positive correlation between nPAS\% and dysphagia. Thus, we analyzed the association between nPAS\% and other radiographic parameters. The correlation between dPIA and its potential influencing factors was also evaluated. A multiple regression analysis was used to examine the radiographic variables that affected dnPAS\%. A p-value less than 0.05 indicated a significant difference. Power analysis (using 0.8 power and 0.05 significance) was used to calculate the required sample size.

\section{Results}

Ninety-eight patients matched to the criteria were enrolled in this study, including 47 male patients and 51 female patients. The average age at the time of surgery was 50.7 years, and the mean follow-up period was 56.4 months (ranging from 12 to 110 months). Of all the patients, 26 experienced postoperative dysphagia, and the rate of occurrence was $26.5 \%$. Among the 26 patients, only two experienced total relief of dysphagia during follow-up. However, dysphagia symptoms for the remaining 24 patients persisted until the final follow-up. Seven of these patients experienced partial relief. None of the 26 dysphagia patients underwent revision surgery.
Dysphagia information of these patients at postoperative and final follow-up is shown in Table 1.

\section{Radiographic results}

The inter- and intraobserver intraclass correlation coefficient values for the radiographic parameters were all more than 0.9 , demonstrating excellent repeatability.

We subdivided the patients into a dysphagia group and a nondysphagia group. Between the two groups, all parameters preoperatively were at a similar baseline except PIA. The preoperative PIA in the dysphagia group was significantly higher than that in the nondysphagia group. When comparing the postoperative values, we found that postoperative O-C2a, O-EAa, PIA and nPAS were significantly lower in the dysphagia group than in the nondysphagia group. However, C2-7a was significantly higher in the dysphagia group. In the dysphagia group, significant decreases were found in ADI, O-C2a, O-EAa, PIA and nPAS from preoperative to postoperative. In the nondysphagia group, we detected significant increases in $\mathrm{O}-\mathrm{C} 2 \mathrm{a}$ and decreases in ADI, C2Ta and C27a. The changes in O-C2a, O-EAa, C2-7a, PIA and nPAS\% between the two groups all showed significant differences. Data are shown and compared in Table 2.

Simple linear regression showed that dnPAS\%, dOC2a, dO-EAa, dC2-7a and dPIA all had significant 
Table 1 Dysphagia information of patients in dysphagia group

\begin{tabular}{|c|c|c|c|c|c|c|c|}
\hline No. & $\begin{array}{l}\text { Grade of dysphagia } \\
\text { (postoperative) }\end{array}$ & $\begin{array}{l}\text { Duration } \\
\text { (mo) }\end{array}$ & $\begin{array}{l}\text { Grade of dysphagia } \\
\text { (final follow-up) }\end{array}$ & No. & $\begin{array}{l}\text { Grade of dysphagia } \\
\text { (postoperative) }\end{array}$ & $\begin{array}{l}\text { Duration } \\
\text { (mo) }\end{array}$ & $\begin{array}{l}\text { Grade of dysphagia } \\
\text { (final follow-up) }\end{array}$ \\
\hline 1 & Moderate & 110 & Mild & 14 & Moderate & 43 & Mild \\
\hline 2 & Severe & 108 & Severe & 15 & Moderate & 41 & Moderate \\
\hline 3 & Mild & 98 & Mild & 16 & Mild & 41 & Mild \\
\hline 4 & Mild & 8 (relief) & None & 17 & Mild & 40 & Mild \\
\hline 5 & Moderate & 74 & Moderate & 18 & Moderate & 24 (relief) & None \\
\hline 6 & Mild & 74 & Mild & 19 & Mild & 35 & Mild \\
\hline 7 & Severe & 70 & Severe & 20 & Severe & 33 & Moderate \\
\hline 8 & Mild & 68 & Mild & 21 & Mild & 20 & Mild \\
\hline 9 & Mild & 66 & Mild & 22 & Mild & 18 & Mild \\
\hline 10 & Moderate & 66 & Mild & 23 & Moderate & 16 & Mild \\
\hline 11 & Severe & 55 & Moderate & 24 & Moderate & 13 & Mild \\
\hline 12 & Mild & 48 & Mild & 25 & Moderate & 12 & Moderate \\
\hline 13 & Mild & 46 & Mild & 26 & Mild & 12 & Mild \\
\hline
\end{tabular}

Table 2 Patients' radiographic outcomes (mean \pm SD)

\begin{tabular}{|c|c|c|c|}
\hline & $\begin{array}{l}\text { Dysphagia } \\
\text { (26 patients) }\end{array}$ & $\begin{array}{l}\text { Without Dysphagia } \\
\text { (72 patients) }\end{array}$ & $P$ value \\
\hline Pre ADI & $0.60 \pm 0.26 \mathrm{~cm}$ & $0.59 \pm 0.35 \mathrm{~cm}$ & 0.554 \\
\hline Pre $0-C 2 a$ & $9.58 \pm 10.21$ & $4.04 \pm 16.01$ & 0.191 \\
\hline Pre O-EAa & $104.32 \pm 7.44$ & $101.33 \pm 7.94$ & 0.169 \\
\hline Pre C2Ta & $94.73 \pm 13.52$ & $97.42 \pm 17.72$ & 0.504 \\
\hline Pre C2-7a & $20.48 \pm 14.06$ & $25.67 \pm 18.50$ & 0.211 \\
\hline Pre PIA & $96.80 \pm 8.00$ & $90.06 \pm 11.42$ & $0.005^{*}$ \\
\hline Pre nPAS & $1.25 \pm 0.45 \mathrm{~cm}$ & $1.34 \pm 0.48 \mathrm{~cm}$ & 0.316 \\
\hline Post ADI & $0.33 \pm 0.14 \mathrm{~cm}^{\#}$ & $0.33 \pm 0.12 \mathrm{~cm}^{\#}$ & 0.971 \\
\hline Post O-C2a & $0.07 \pm 9.12^{\#}$ & $7.86 \pm 13.96^{\#}$ & $0.005^{*}$ \\
\hline Post O-EAa & $94.21 \pm 7.87^{\#}$ & $101.42 \pm 6.44$ & $0.000^{*}$ \\
\hline Post C2Ta & $94.13 \pm 12.31$ & $93.56 \pm 14.34^{\#}$ & 0.863 \\
\hline Post C2-7a & $24.60 \pm 13.27$ & $18.00 \pm 15.58^{\#}$ & $0.038^{*}$ \\
\hline Post PIA & $81.85 \pm 5.51^{\#}$ & $91.28 \pm 10.50$ & $0.000^{*}$ \\
\hline Post nPAS & $0.69 \pm 0.31 \mathrm{~cm}^{\#}$ & $1.43 \pm 0.50 \mathrm{~cm}$ & $0.000^{*}$ \\
\hline dADI & $-0.27 \pm 0.22 \mathrm{~cm}$ & $-0.26 \pm 0.28 \mathrm{~cm}$ & 0.621 \\
\hline do-C2a & $-9.51 \pm 5.18$ & $3.82 \pm 11.71$ & $0.000^{*}$ \\
\hline dO-EAa & $-10.11 \pm 5.12$ & $0.10 \pm 7.15$ & $0.000^{*}$ \\
\hline $\mathrm{dC2} \mathrm{Ta}$ & $-0.61 \pm 5.88$ & $-3.86 \pm 11.37$ & 0.159 \\
\hline dC2-7a & $4.11 \pm 13.93$ & $-7.66 \pm 13.57$ & $0.001^{*}$ \\
\hline dPIA & $-13.95 \pm 6.87$ & $1.22 \pm 11.12$ & $0.000^{*}$ \\
\hline dnPAS\% & $-43.84 \pm 16.33 \%$ & $16.90 \pm 59.10 \%$ & $0.000^{*}$ \\
\hline
\end{tabular}

SD Standard deviation, Pre Preoperative, ADI Atlas-dens interval, O-C2a O-C2 angle, O-EAa Occipital and external acoustic meatus to axis angle, C2Ta C2 tilting angle, PIA Pharyngeal inlet angle, nPAS The narrowest oropharyngeal airway space, Post Postoperative, $d$ The difference between pre- and postoperative values, $d n P A S \%$ (postoperative nPAS- preoperative nPAS)/ preoperative $\mathrm{nPAS}{ }^{*} p<0.05$ between groups; ${ }^{*} p<0.05$ comparing with preoperative data correlations with the presence of postoperative dysphagia. The scatter diagram and data are shown in Fig. 2.

When analyzing the relationship between $\mathrm{dnPAS} \%$ and other parameters, we found significant correlations in four of them, including dO-C2a, dO-EAa, dC2-7 and dPIA. The $\mathrm{R}^{2}$ values of dO-EAa $\left(\mathrm{R}^{2}=0.457\right)$ and dPIA $\left(R^{2}=0.423\right)$ were similar, and both were above 0.4 , which demonstrated a strong correlation with dnPAS\%. The scatter diagram and data are shown in Fig. 3. Furthermore, multiple regression analysis showed that among these four parameters, dO-EAa and dPIA were the only significant predictors for dnPAS\% (dO-EAa: $\beta=0.435, p=$ 0.000; dPIA: $\beta=0.285, p=0.029$ ) (Table 3).

Based on the calculated parameters, the required sample size (using 0.8 power and 0.05 significance) for our study was 53 (14 patients in the dysphagia group and 39 in the nondysphagia group). In addition, the statistical power for our sample size was 0.97 . This showed that our study was powered to detect the difference in parameters affecting postoperative dysphagia.

The radiographic presentation of a patient from the dysphagia group is shown in Fig. 4.

\section{Discussion}

OCF has become a conventional and effective operation method for treating instability of the craniocervical junction caused by trauma, tumors, congenital deformities, rheumatoid arthritis, and infection, et al. [10, 14-16]. It has been reported that $1 / 6$ to $1 / 4$ of patients who underwent OCF experienced dysphagia postoperatively, and only a small proportion of them experienced total symptom relief during follow-up [1-4]. As shown in our study, 


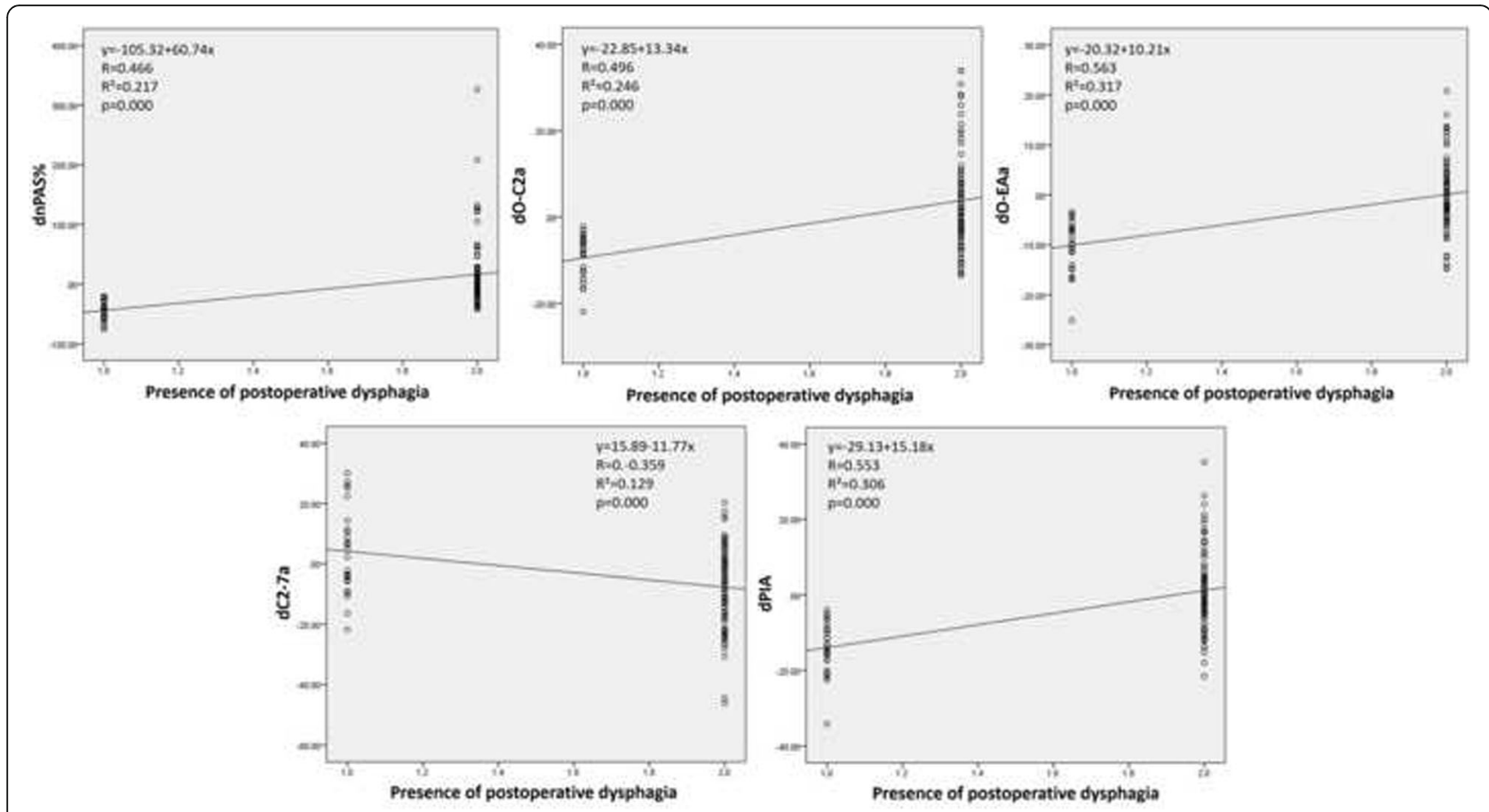

Fig. 2 Scatter diagrams between the changes in pre- and postoperative radiographic parameters and the presence of dysphagia. $d O-E A a(R 2=$ $0.317)$ and $d P I A(R 2=0.306)$ had a superior correlation with postoperative dysphagia compared with dnPAS\%, dO-C2a and dC2-7a
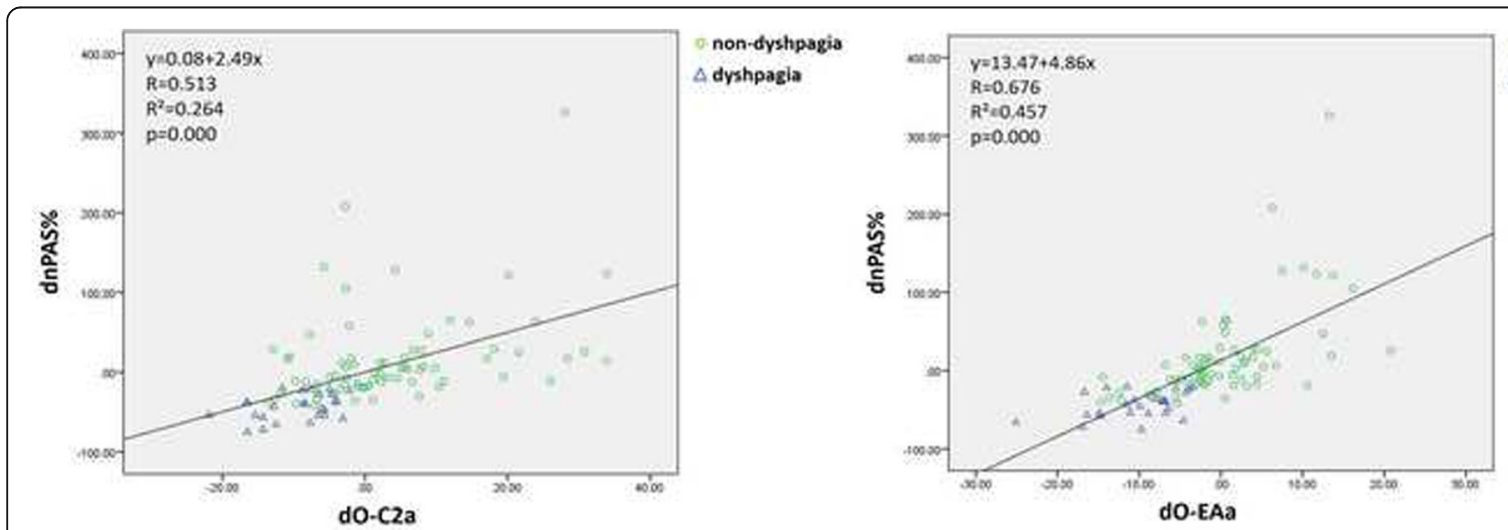

Eon-dyshpagia

$\triangle$ drshpagia
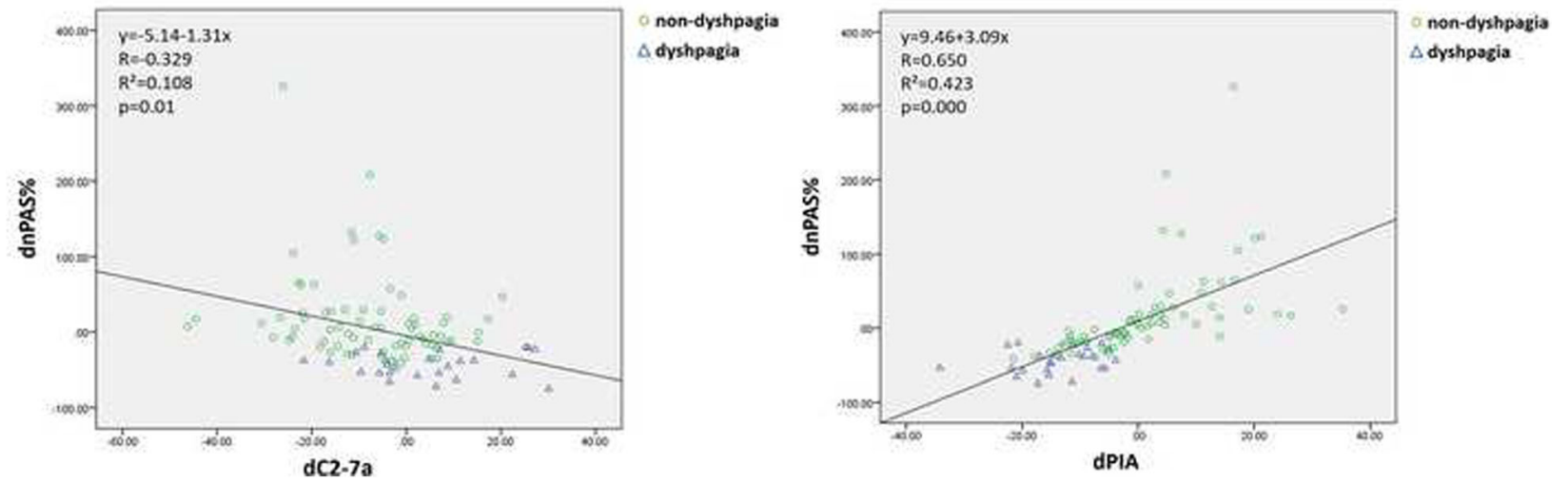

Fig. 3 Scatter diagrams between dnPAS\% and predictive radiographic parameters. dO-EAa $(R 2=0.457)$ and dPIA (R2=0.423) both have obvious correlations with dnPAS\%, superior to those with dO-C2a and dC2-7a 
Table 3 Multivariate linear regression analysis for the influence of predictors on dnPAS\%

\begin{tabular}{|c|c|c|c|c|c|c|c|}
\hline \multirow[b]{2}{*}{ Predictors for dnPAS\% } & \multicolumn{2}{|c|}{$\begin{array}{l}\text { Unstandardized } \\
\text { coefficient }\end{array}$} & \multirow{2}{*}{$\begin{array}{l}\text { Standardized } \\
\text { coefficient } \\
\beta\end{array}$} & \multicolumn{4}{|c|}{ 95\% Confidence interval } \\
\hline & $\bar{B}$ & SE & & Lower limit & Upper limit & $\mathrm{t}$ value & $p$ \\
\hline dO-C2a & 0.200 & 0.531 & 0.041 & -0.854 & 1.254 & 0.376 & 0.708 \\
\hline dO-EAa & 3.126 & 0.729 & 0.435 & 1.678 & 4.574 & 4.287 & $0.000^{*}$ \\
\hline$d C 2-7 a$ & -0.256 & 0.325 & -0.064 & -0.902 & 0.390 & -0.787 & 0.433 \\
\hline dPIA & 1.358 & 0.613 & 0.285 & 0.141 & 2.574 & 2.216 & $0.029^{*}$ \\
\hline Constant & 11.528 & 4.797 & & 2.003 & 21.054 & 2.403 & $0.018^{*}$ \\
\hline
\end{tabular}

dnPAS\%, [postoperative narrowest oropharyngeal airway space (nPAS) - preoperative nPAS]/preoperative nPAS; dO-C2a, the difference between pre- and postoperative O-C2 angle; dO-EAa, the difference between pre- and postoperative occipital and external acoustic meatus to axis angle; dC2-7a, the difference between pre- and postoperative C2-7 angle; $\mathrm{dPIA}$, the difference between pre- and postoperative pharyngeal inlet angleAdjust $\mathrm{R}^{2}=0.501, p<0.001^{*}$, Indicates statistically significant differences $(p<0.05)$

only two of the 26 dysphagia patients experienced total relief. Thus, risk factors for dysphagia after OCF have been widely discussed $[1,10,11,17]$. In recent years, how to avoid postoperative dysphagia by adjusting the occipitocervical angle has been widely studied $[4,6-8,10,11]$.

$\mathrm{O}-\mathrm{C} 2 \mathrm{a}$ has been proven to have predictive ability for post-OCF dysphagia $[1,9,10,18]$. However, in Izeki et al.'s study, reduction of anterior atlantoaxial subluxation (AAS) caused a decrease in nPAS despite an increase in $\mathrm{O}-\mathrm{C} 2 \mathrm{a}$ because the atlas shifted posteriorly [11]. Later, Morizane et al. promoted a new parameter, O-EAa, which could reflect not only the change in atlantooccipital angle but also the translational motion of the atlas [7]. They proved that O-EAa was superior to OC2a in predicting changes in nPAS for AAS patients [19]. In our previous study, we evaluated this angle for all OCF patients and found that the predictive ability of O-EAa surpassed that of O-C2a in postoperative dysphagia [4]. However, during the measurement, we found that the external acoustic meatus was unreadable for some patients. In these cases, the position of the external acoustic meatus was estimated by display of the pinna, which would increase the measurement error. PIA, formed by McGregor's line and the line linking the center of the $\mathrm{C} 1$ anterior arch and the apex of the cervical sagittal curvature, was first promoted by Kaneyama et al. and has been proven to be effective in predicting postoperative dysphagia in patients who underwent occipitospinal fusion. Meanwhile, its anatomic structures can be easily identified on radiographic images [3]. Thus, in our study, we evaluated the predictive ability of PIA in postoperative dysphagia for patients who underwent OCF.

When comparing the radiographic parameters, we found that postoperative O-C2a, O-EAa, PIA and nPAS all had significant decreases in the dysphagia group compared with the nondysphagia group, while C2-7 had a significant increase. When analyzing the linear correlation, the changes in O-C2a, PIA and O-EAa had significant correlations with both dnPAS and the presence of dysphagia. Furthermore, the $\mathrm{R}^{2}$ values of O-EAa and
PIA were similar and higher than that of O-C2a. The results of multiple regression analysis showed that among all the radiographic parameters, only O-EAa and PIA were significant risk factors for changes in nPAS. Considering all the results, we thought that a decrease in PIA would cause oropharyngeal stenosis and may result in postoperative dysphagia. In addition, PIA has a similar predictive effect as O-EAa in the change in nPAS and the occurrence of postoperative dysphagia.

Previously, Kaneyama et al. considered that PIA was affected by the position of the atlas and cervical sagittal curvature [3]. Thus, in our study, we analyzed the relationships of dADI, dO-C2a, and dC2-7a with dPIA. Similar to their findings, the results of our study showed that dPIA had significant correlations with $\mathrm{dO}-\mathrm{C} 2 \mathrm{a}$ and $\mathrm{dC} 2-7 \mathrm{a}$, which is either an increase in $\mathrm{O}-\mathrm{C} 2 \mathrm{a}$ or a decrease in C2-7a could lead to an increase in PIA. Furthermore, the correlation of dO-C2a \& dPIA was much higher than that of dC2-7 \& dPIA. The scatter diagram and data are shown in Fig. 5. However, the change in ADI had no correlation with the change in PIA ( $p=$ $0.497, \mathrm{R}=0.069$ ). We assumed this may be because a reduction in AAS could not only lead to a posterior shift of the atlas but also an increase in O-C2a. The changes had a complicated effect on PIA.

After reviewing the radiographic data, we found that 35 of the 98 patients had a dPIA $\geq 0$. All 35 patients had no postoperative dysphagia, and only two of them had a slight decrease in nPAS, which demonstrated that an increase in PIA could help prevent the occurrence of dysphagia. Moreover, we found that the preoperative PIA in the dysphagia group was significantly higher than that in the nondysphagia group. For patients who had a high preoperative PIA, preserving or increasing PIA during surgery sometimes seemed to be difficult due to requirements such as achieving adequate reduction and preserving a horizontal gaze. Thus, for these patients, whether there would be a threshold PIA for preventing postoperative dysphagia was analyzed. 


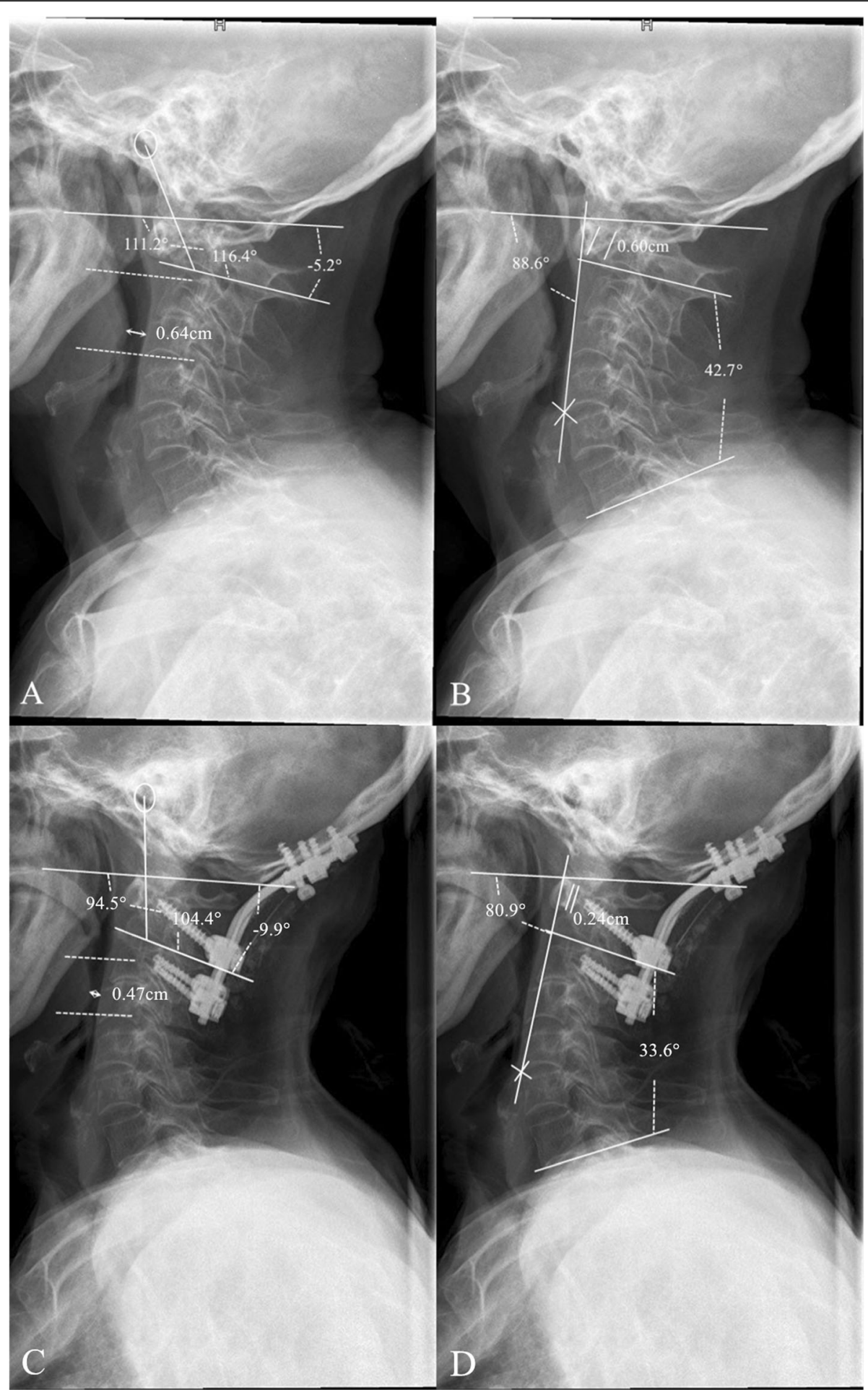

Fig. 4 Radiographic presentation of an atlantoaxial dislocation patient suffering postoperative dysphagia. a The preoperative values of O-EAa and O-C2a were $111.2^{\circ}$ and $-5.2^{\circ}$, respectively, and nPAS was $0.64 \mathrm{~cm}$. bThe preoperative values of C2-7a and PIA were $88.6^{\circ}$ and $42.7^{\circ}$, respectively. $\mathrm{ADI}$ was $0.60 \mathrm{~cm}$. $\mathbf{c}$ and $\mathbf{d}$ Postoperative measurement of radiographic parameters. On postoperative measurements, O-EAa, O-C2a and C2-7a decreased to $94.5^{\circ},-9.9^{\circ}$ and $33.6^{\circ}$, respectively, and PIA decreased to $80.9^{\circ}$. Atlantoaxial reduction was achieved. The oropharyngeal space decreased significantly to $0.47 \mathrm{~cm}$ and experienced postoperative dysphagia 

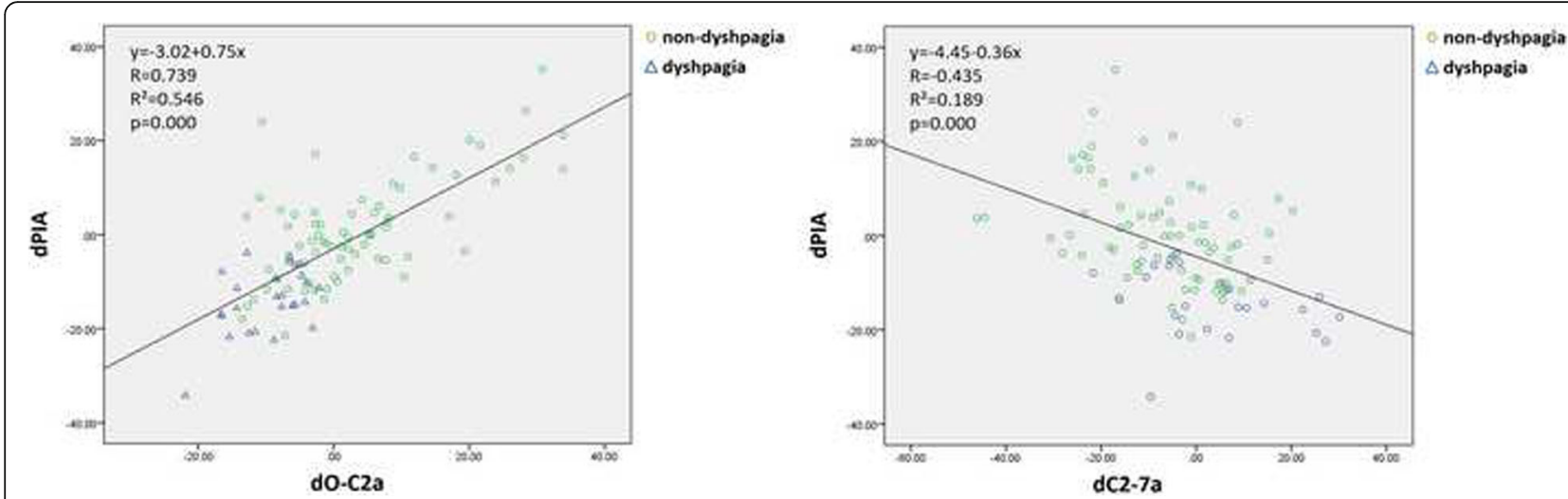

Fig. 5 Scatter diagrams between dPIA and predictive radiographic parameters. dO-C2a and dC2-7a could lead to an increase in PIA. In addition, the increase in $\mathrm{O}-\mathrm{C} 2 \mathrm{a}(\mathrm{R} 2=0.546)$ had a superior correlation with the increase in PIA compared with the decrease in $C 2-7 \mathrm{a}(\mathrm{R} 2=0.189)$

In Kaneyama et al.'s study, no patients experienced dysphagia with a postoperative PIA of no less than 90 . They considered PIA $\geq 90$ to be a threshold for preventing postoperative dysphagia [3]. In the present study, the sensitivity and specificity of PIA $<90^{\circ}$ in predicting postoperative dysphagia were $88.5 \%$ (23/26) and 58.3\% (42/ 72), respectively. Only three patients with postoperative PIA over 90 experienced dysphagia. The PIAs of these three patients were all close to 90 (90.4, 90.4 and 91.3) and were within the measurement error. Thus, we assume that preserving a PIA over 90 could greatly reduce the occurrence of postoperative dysphagia.

In summary, we believe that for all patients, adjusting a $\mathrm{dPIA} \geq 0$ during the operation would avert postoperative dysphagia, and for those with a high preoperative PIA in whom a decrease is inevitable, postoperative PIA should be maintained over 90 . The surgeon could alter the PIA by adjusting the patient's head position for an increased $\mathrm{O}-\mathrm{C} 2 \mathrm{a}$ (through bending the rods or shortening the interval among fixed segments) before the final fixation during surgery.

One major concern about using PIA as the predictor for postoperative dysphagia in OCF patients is that the lower cervical spine was unfixed. The mobile lower cervical spine would change the intraoperative adjustment of PIA. However, theoretically, if we extend the upper cervical spine to increase the O-C2a and PIA before final fixation during surgery, flexion at the lower cervical spine would occur postoperatively to compensate for the horizontal gaze. In this condition, $\mathrm{O}-\mathrm{C} 2 \mathrm{a}$ maintained while $\mathrm{C} 2-7 \mathrm{a}$ decreased, resulting in an increase in the PIA. Therefore, we thought that PIA could be used as a predictor for avoiding postoperative dysphagia in patients who underwent OCF. However, this hypothesis should be proven in future studies.

There are some shortcomings in this study. First, this was a retrospective study; the data collected may be less accurate and complete than prospective studies. Second, the dysphagia of patients was assessed by inquiry, and there was a lack of radiographic evidence, such as the barium swallow test.

\section{Conclusions}

Postoperative dysphagia is a common complication after OCF and affects patients' daily living. The present study demonstrated that PIA had a similar predictive effect as $\mathrm{O}-\mathrm{EAa}$ and could be used as a predictor for postoperative dysphagia in patients undergoing OCF. Adjusting intraoperative PIA over preoperative PIA could help avoid postOCF dysphagia. For those with an inevitable decrease in PIA, preserving the intraoperative PIA more than 90 would to a great extent avert postoperative dysphagia.

\section{Abbreviations}

OCF: Occipitocervical fusion; O-C2a: O-C2 angle; O-EAa: Occipital and external acoustic meatus to axis angle; C2Ta: C2 tilting angle; PIA: Pharyngeal inlet angle; ADI: Atlas-dens interval; C2-7a: C2-7 angle; nPAS: Narrowest oropharyngeal airway space; AAS: Anterior atlantoaxial subluxation

\section{Acknowledgements}

We would like to express our thanks to the patients who generously agreed to be interviewed for this study.

\section{Authors' contributions}

Drafting of the manuscript: LNW. Study concept and design: LNW, BWH, YMS, LML and XY. Acquisition of data: LNW, BWH, XY. Analysis and interpretation of data: LNW, BWH, LW, CGZ, XY. Supervision: YMS and LML. The authors read and approved the manuscript.

\section{Funding}

This work was funded by Post-Doctor Research Project, West Chnia Hospital, Sichuan University (2019HXBH007) and National Key Research and Development Program of China (2016YFC1102004).

\section{Availability of data and materials}

The data that support the findings of this study are available from the corresponding author $\mathrm{XY}$, upon reasonable request. The data are not publicly available due to their containing information that could compromise the privacy of research participants.

\section{Ethics approval and consent to participate}

This study was approved by local Medical Ethics Committee and informed consent was obtained from all individual participants included in the study in written forms. 


\section{Consent for publication}

Not applicable.

\section{Competing interests}

The authors declare that they have no conflict of interest.

Received: 28 September 2020 Accepted: 26 December 2020 Published online: 09 January 2021

\section{References}

1. Miyata M, Neo M, Fujibayashi S, Ito H, Takemoto M, Nakamura T. O-C2 Angle as a predictor of dyspnea and/or dysphagia after occipitocervical fusion. Spine. 2009;34(2):184-8.

2. Matsuyama Y, Kawakami N, Yoshihara H, Tsuji T, Kamiya M, Yukawa Y, et al. Long-term results of occipitothoracic fusion surgery in RA patients with destruction of the cervical spine. J Spinal Disord Tech. 2005; 18(Suppl.):101-6.

3. Kaneyama S, Sumi M, Takabatake M, Kasahara K, Kanemura A, Hirata H, et al. The prediction and prevention of dysphagia after occipitospinal fusion by use of the S-line (swallowing Line). Spine. 2017:42:718-25.

4. Wang LN, Hu BW, Song YM, Liu LM, Zhou CG, Wang L, et al. Predictive abilities of O-C2a and O-EAa for the development of postoperative dysphagia in patients undergoing occipitocervical fusion. Spine J. 2020; 20(5):745-53.

5. Kaneyama S, Sumi M, Kasahara K, Kanemura A, Takabatake M, Yano T. Dysphagia after occipitothoracic fusion is caused by direct compression of oropharyngeal space due to anterior protrusion of mid-cervical spine. J Spinal Disord Tech. 2014;30(7):314-20.

6. Tagawa T, Akeda K, Asanuma Y, Miyabe M, Arisaka H, Furuya M, et al. Upper airway obstruction associated with flexed cervical position after posterior occipitocervical fusion. J Anesth. 2011;25(1):120-2.

7. Morizane K, Takemoto M, Neo M, Fujibayashi S, Otsuki B, Kawata T, et al. Occipital and external acoustic meatus to axis angle as a predictor of the oropharyngeal space in healthy volunteers: a novel parameter for craniocervical junction alignment. Spine J. 2018;18:811-7.

8. Nagashima S, Nagae M, Arai $Y$, Tonomura H, Takatori R, Sukenari T, et al. A new method of measuring the occipitocervical angle that could be applied as an intraoperative indicator during occipitocervical fusion. Clin Spine Surg. 2017;30(7):E981-7

9. Yoshida M, Neo M, Fujibayashi S, Nakamura T, et al. Upper-Airway obstruction after short posterior occipitocervical fusion in a flexed position. Spine. 2007;32(8):E267-70.

10. Meng Y, Wu TK, Liu ZY, Wen D, Rong $X$, Chen $H$, et al. The impact of the difference in $\mathrm{O}-\mathrm{C} 2$ angle in the development of dysphagia after occipitocervical fusion: a simulation study in normal volunteers combined with a case-control study. Spine J. 2018;18:1388-97.

11. Izeki M, Neo M, Ito H, Nagai K, Ishizaki T, Okamoto T, et al. Reduction of atlantoaxial subluxation causes airway stenosis. Spine. 2013;38(9): E513-20.

12. Joaquim AF, Murar J, Savage JW, Patel AA. Dysphagia after anterior cervical spine surgery: a systematic review of potential preventative measures. Spine J. 2014;14(9):2246-60.

13. Bazaz R, Lee MJ, Yoo JU. Incidence of dysphagia after anterior cervical spine surgery: a prospective study. Spine. 2002;27(22):2453-8.

14. Zuckerman SL, Kreines F, Powers A, lorgulescu JB, Elder JB, Bilsky MH, et al. Stabilization of tumor-associated craniovertebral junction instability: indications, operative variables, and outcomes. Neurosurgery. 2017:81:251-8

15. Garrido BJ, Sasso RC. Occipitocervical fusion. Orthop Clin North Am. 2012; 43(1):1-9.

16. Qureshi M, Afzal W, Khalique A, Pasha I, Aebi M. Tuberculosis of the craniovertebral junction. Eur Spine J. 2012;22(S4):612-7.

17. Wattenmaker I, Conception M, Hibberd P, Lipson S. Upper-airway obstruction and perioperative management of the airway in patients managed with posterior operations on the cervical spine for rheumatoid arthritis. J Bone Joint Surg Am. 1994;76:360-5.

18. Wang $\mathrm{X}$, Chou D, Jian F. Influence of postoperative O-C2 angle on the development of dysphagia after occipitocervical fusion surgery: results from a retrospective analysis and prospective validation. World Neurosurg. 2018; 116:e595-601
19. Morizane K, Takemoto M, Neo M, Fujibayashi S, Otsuki B, Tanida S, et al. Occipital and external acoustic meatus to axis angle: a useful predictor of oropharyngeal space in rheumatoid arthritis patients with atlantoaxial subluxation. J Neurosurg Spine. 2019;31(4):534-41. Epub ahead of print.

\section{Publisher's Note}

Springer Nature remains neutral with regard to jurisdictional claims in published maps and institutional affiliations.
Ready to submit your research? Choose BMC and benefit from:

- fast, convenient online submission

- thorough peer review by experienced researchers in your field

- rapid publication on acceptance

- support for research data, including large and complex data types

- gold Open Access which fosters wider collaboration and increased citations

- maximum visibility for your research: over $100 \mathrm{M}$ website views per year

At BMC, research is always in progress.

Learn more biomedcentral.com/submissions 